\title{
Network Coding-based Maximum Lifetime Algorithm for Sliding Window in WSNs
}

\author{
Baolin Sun $^{1 *}$, Chao Gui ${ }^{1}$, Ying Song ${ }^{1 *}$, and Hua Chen ${ }^{2}$ \\ ${ }^{1}$ School of Information and Communication Engineering, Hubei University of Economics \\ Wuhan 430205, China \\ [e-mail: blsun@163.com,gui_chao@126.com, prisong@163.com] \\ ${ }^{2}$ Department of Public Basic Course, Wuhan Technology and Business University \\ Wuhan 430065, China \\ [e-mail: qiuchen_1022@163.com] \\ *Corresponding author: Baolin Sun, Ying Song
}

Received August 14, 2017; revised March 18, 2018; accepted August 14, 2018;

published March 312019

\begin{abstract}
Network coding (NC) is a promising technology that can improve available bandwidth and packet throughput in wireless sensor networks (WSNs). Sliding window is an improved technology of NC, which is a supplement of TCP/IP technology and can improve data throughput and network lifetime on WSNs. This paper proposes a network coding-based maximum lifetime algorithm for sliding window in WSNs (NC-MLSW) which improves the throughput and network lifetime in WSN. The packets on the source node are sent on the WSNs. The intermediate node encodes the received original packet and forwards the newly encoded packet to the next node. Finally, the destination node decodes the received encoded data packet and recovers the original packet. The performance of the NC-MLSW algorithm is studied using NS2 simulation software and the network packet throughput, network lifetime and data packet loss rate were evaluated. The simulations experiment results show that the NC-MLSW algorithm can obviously improve the network packet throughput and network lifetime.
\end{abstract}

Keywords: WSN, sliding window, network coding, network lifetime, network throughput

This work was supported by the National Natural Science Foundations of China (Grants no. 61572012), The Natural Science Foundation of Hubei Province of China (No. 2018CFB661). 


\section{Introduction}

W ireless sensor network (WSN) is composed of many distributed, randomly deployed, small sensors, disaster relief and sensor nodes with communication capability. Typically, WSN consists of many wireless sensor nodes that perform special calculations and specific tasks [1-6]. Since the sensor nodes do not need to be charged or replaced with batteries, the WSN nodes are usually energy constrained. Over the past few years, the processing power of wireless sensor nodes has improved significantly, while the battery power of these nodes has not. How to improve the packet transmission efficiency of WSN, reduce the packet loss rate of WSN, and maximize the lifetime is still a main challenge.

Sliding window is a common technique to increase packet transmission efficiency in WSNs. In dense wireless networks, duty cycle based wireless sensor nodes can improve network life. In addition, in the deployment process of wireless sensor networks, the density of sensor nodes may remain at a high level, so that the network can cover a certain range and make connectivity of nodes better. However, in dense wireless sensor networks, wireless nodes consume more energy due to redundant data packet transmission and reception. Traditional data transmission technology mainly uses data correlation to improve the reliability of data packet transmission and enhance the wireless network traffic.

Ahlswede et al. [1] studied the energy consumption and network throughput of multicast transmission in wireless networks and concluded that network coding could solve such problems in a better way. However, they did not give specific NC implementation scheme. In the last decade, the network coding algorithm has been well implemented in wireless networks. However, in the WSN system, the complexity of network coding algorithm is low because the intermediate node limits the network coding operation to the binary limited domain and all the coding operations XOR-ing of bits [1-6]. In this research work, network coding algorithm is used to improve the network data throughput, energy efficiency and network life of WSNs.

In literature [2], the author discussed the NC framework and compared comprehensive NC research and analysis on two types of information transmission: static data and dynamic video data transmission over WSNs. The main methods to implement network coding are Random Network Coding, Linear Network Coding, Cross-layer Network Coding and so on. Many of these network coding methods are related to multicast transmission, multipath transmission, energy efficiency and other transmission technologies. NC can provide excellent data transmission advantages through mixed data packets in wireless networks. These advantages are especially introduced in the transmission and application of combined data packets. Network coding has a wide application field, and has its application basis in different systems. Yeung [3] emphasized the application of network coding when discussing the development and classification of network coding, and put forward a seminal paper that network coding had a good application. Li et al. [4] addressed the problem of robust network coding. Combining techniques of robust network data packets, linear algebra and randomization, a polynomial time algorithm for robust linear multicast network coding is constructed, which can reduce the complexity of network coding effectively. Aktas et al. [5] proposed a simple and effective network encoding and decoding algorithm for multicast and unicast wireless networks in view of multicast and unicast data transmission technologies in wireless networks, which can improve the data transmission efficiency of multicast and unicast data better. Kagi et al. [6] proposed a multipath routing algorithm using multiple nodes disjoint routing to solve the 
problem of network coding on relay nodes in wireless network, which can improve the processing capacity of relay nodes.

In our previous research, we proposed a multipath routing network coding algorithm in WSN (NC-WSN) [7], which can improve the throughput of data packet and the reliability of data packet transmission, and achieve the load balancing ability of data packet transmission better. In literature [8], we proposed a sliding window network coding algorithm in MANETs (SWNCM). The SWNCM algorithm preserves the degree distribution of coded data packets by packets and recombining the intermediate nodes. SWNCM controls the decoding complexity of each sliding window independently of the received data packets, and can quickly restore the original data packets after receiving the encoded data packets.

Under the traditional packet coding method, the sending rate of these receiving nodes is zero. However, with deterministic network coding, it is possible for the receiver to decode all the data packets and recover the original data packets even though the receiver cannot receive all the data packets transmitted by the sender. The major contributions are as follows:

- Development of a maximum lifetime algorithm using network coding to improve the network data packet throughput, and network lifetime in WSNs.

- Mathematical analysis of network coding-based maximum lifetime and sliding window and for a WSN with NC-MLSW mechanism.

- Performance of the data packet throughput, network lifetime of a sliding window based WSN and NC-MLSW mechanism.

The remainder of this paper is as follows. Section 2 discusses the some related work. Section 3 describes an adaptive length sliding window models in WSN. Section 4 discusses network lifetime using network coding. Section 5 demonstrates mathematical analyses. Some simulating results are providing in section 6 . Finally, the paper concludes in section 7.

\section{Related Work}

The research of network coding is mainly to prevent the network performance loss caused by poor link quality of wireless link. Rout et al. [9] analyzed the ratio between the time when wireless sensor nodes are active and the total time when they are active/inactive, and proposed the utilization of duty ratio and network coding to improve the data packet transmission rate and network lifetime of WSNs. In the bottleneck area of WSNs, Lee et al. [10] proposed an network coding and duty cycling aware energy-saving algorithm, which can extend the life of WSNs. In this algorithm, the packet forwarding in the coding node adopts random linear network coding mechanism, thus improving the energy efficiency and reliability of the nodes in the bottleneck region.

$\mathrm{Gu}$ et al. [11] proposes a network coding-aware hybrid wireless mesh routing protocol (CAHWMP) for WMN. In the CAHWMP protocol, the author design a coding criterion based on data streams to devise an algorithm for actively detecting coding opportunities during path discovery. Chen et al. [12] proposed a sliding window scheme to construct cyclic and quasi-cyclic data block coding algorithm ( $q$-ary LDPC) in a small fields. This construction algorithm is carried out using a predefined sliding window, which actually handles the general mapping from the original data packet to the data packet under specific parameters. Wu et al. [13] analyzed the characteristics of wireless network model and network coding scheme, and proposed a cooperative multicast protocol based on sliding window network coding technology (MWNCast). In literature [14], the real-time decodable network coding is analyzed and extended, so that each data packet can meet the delay constraint, but the decoding performance is optimal only when the receiver does not exceed three packets. 
During recent years, the energy-efficient data transmission in WSNs has been a hot topic among both industrial and academic communities [15, 16, 17, 20]. Energy-efficient designs are essential for some WSNs, especially for networks with energy-constraint sensors. It is interesting to investigate how the NC technology improves the energy efficiency in wireless networks. Liu et al. [15] proposed an optimal energy saving power distribution algorithm (OE-PA), which minimizes total energy consumption per bit under the sum rate constraint and interference power threshold (IPT) constraint. The OE-PA protocol has superiority in energy efficiency and network lifetime performance over conventional direct transmission protocol. Tian et al. [16] proposed a new efficient and energy-saving packet coding backup algorithm based on network coding. This algorithm can reduce the total energy consumption of all sensor nodes in WSN to the lowest level according to the demand of data stream transmission. Fiandrotti et al. [17] studied the packet degree distribution of network codes in the process of packet coding, recombination and decoding, and proposed a new Band Codes (BC). BC is a random network coding scheme on GF(2), with low decoding complexity, structure control degree distribution and feature limitation. Therefore, BC can be well applied to NC even in the case of energy limitation. Zhou et al. [18] analyzed the node energy saving mechanism in wireless sensor network and proposed an improved node energy saving compression network coding method, which can better reduce the energy consumption of nodes. Ding et al. [19] studied the coding mechanism, energy consumption and the routing problem of maximizing the coding life cycle of network coding in WSNs, and proposed a traffic-enhanced routing algorithm based on network coding and local energy minimization of single-hop wireless network.

Network coding can be applied to the backward compatible enhanced TCP mechanism to improve the efficiency of data transmission. Lu et al. [20] research the queue state problem of packet coding control strategy in wireless networks, and proposed an enhanced code-aware routing algorithm (OQMCAR) based on queue state and local topology, which can better control the packet data involved in network coding. Wang et al. [21] propose a network coding-based Device-to-Device relay cooperative transmission algorithm. The proposed algorithm can dramatically increase the link achievable rate and network lifetime. Gebotys et al. [22] proposed a sliding window phase-only correlation algorithm for synchronizing the electromagnetic radiation emitted by a complex smartphone that uses a chip cache to run its native code. Stitt et al. [23] proposed a parallel sliding window coding algorithm, which can generate multiple parallel windows equal to the number of inputs provided in each cycle. Tan et al. [24] proposes a hierarchical LMS prediction algorithm, which can significantly improve the convergence rate of the algorithm in the initial state of the algorithm, thus reducing the number of actual packet transmitted to the destination node. Tan et al. [25] proposed an analytical unified model of wireless sensor network nodes with temporal death to solve the problem of node energy acquisition in wireless sensor network. The author analyzed the performance and this model by formulating a series of performance metrics.

In order to improve the performance of wireless sensor network, a sliding window-based network coding algorithm is adopted in this paper. The network packet throughput, network lifetime and packet loss rate are analyzed by simulation software.

\section{Sliding Window Models}

Now we mainly discuss sliding window mechanism and random network coding algorithm. It is not necessary to encode all the packets in one transmission data packet, but only necessary to encode the packets in the same window when using this algorithm. This method simplifies 
the solution process of Gaussian elimination at the receiver node, but need continuous feedback between nodes to determine the packet of data received at the receiver node, so as to delete the sent data from the linear combination of the sender node.

In this paper, undirected network graph $G=(V, E)$ is adopted to model the network of WSN, where $V$ represents the set of sensor nodes and $E$ represents the set of undirected links in the WSN. Each wireless link $e=(i, j) \in E$ indicates that sensor node $i$ can transmit data to sensor node $j$. We assume wireless links are symmetric that if $(i, j) \in E ;(i, j)=(j, i) \in E$ as well.

\subsection{The Sliding Window Model}

The sliding window model is an extension of the insertion-only data packet stream model, in which we compute only the latest element of the function $f$ divided by the data stream. Let us consider the combination of $n$ elements $\left(p_{1}, p_{2}, \ldots, p_{n}\right)$ and coding vector $\left(q_{1}, q_{2}, \ldots, q_{n}\right)$. The definition of a subset of the coding vector $\left(p_{f}, \ldots, p_{e}\right)$. $f$ is the start points of the sliding window, $e$ is the end points of the sliding window. Elements that do not belong to the encoding vector in the encoding window are equal to zero. Let the size of the sliding window be $W=e-f+1$, for $N$ windows, there are $n-W+1$ sliding windows of size $W$. We indicate $w_{w}^{f}$ as the sliding window that has size $W$ and leading edge $f$.

We can define the sliding formula of the current sliding window $\mathrm{SW}_{i}$ as.

$$
\mathrm{SW}_{i}=\alpha \cdot \mathrm{SW}_{i-2}+\beta \cdot \mathrm{SW}_{i-1}
$$

Where $\alpha, \beta(0 \leq \alpha, \beta<1$, and $\alpha+\beta=1)$ is the weight assigned to the latest sliding window weight coefficient. When the window sliding mechanism changes, the current sliding window value $\mathrm{SW}_{i}$ will be updated in the old sliding window $\mathrm{SW}_{i-1}$ and $\mathrm{SW}_{i-2}$, so the current sliding window can be used as the new sliding window.

Consider the data packet stream shown in Fig. 1. As shown in Fig. 1, the sliding window size is 5 . Fig. 1 shows the encoding vector generated by the data packet stream size $N=8$, and three of the $N-W+1=4$ possible encoding windows of size $W=5$. The first sliding window, $\mathrm{SW}_{1}$, consists of the first five data packets. The other two sliding windows, $\mathrm{SW}_{2}$ and $\mathrm{SW}_{3}$, are formed by inserting a new data packet into the previous sliding window and removing the oldest data packet from the old window. The last sliding window that currently comprises the data packet is $\mathrm{SW}_{3}$. Given the threshold of the minimum sliding window, the problem can be defined as the number of data packets to be looked up in the current sliding window. Each sliding window must update the number of packets.

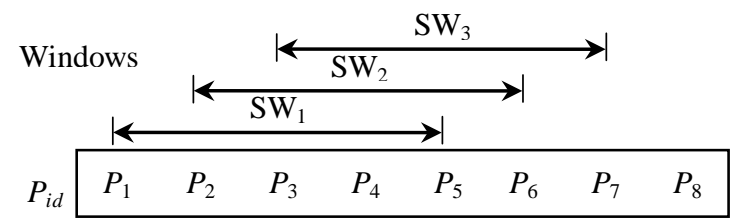

Fig. 1. Data packet stream using sliding window model

\subsection{Smooth Functions}

Let $\boldsymbol{X}$ and $\boldsymbol{R}$ be two finite positive integer sets, a sliding window (SW) is an optimization problem in which the objective function $f: \boldsymbol{X} \rightarrow \boldsymbol{R}$ can be represented as $f=g$ - $h$, where $g, h$ are convex function of SW. The expansion of convex function enables us to make better use of convex analysis and optimization characteristics for performance analysis.

We consider throughout the sliding window SW convex functions:

$$
\min f(x)=g(x)-h(x), x \in \mathbf{R}^{n}
$$


where $g: \mathbf{R}^{n} \rightarrow \mathbf{R}$ and $h: \mathbf{R}^{n} \rightarrow \mathbf{R}$ are monotonic functions. The function $f$ in (2) is called a SW function.

Theorem 1: Consider the function $f$ defined by (2) and the packet sequence $\left\{x_{k}\right\}$ generated by the SW. The following performance characteristics are met:

(1) If $g$ is $\alpha$ - monotonic function and $h$ is $\beta$ - monotonic function, then

$$
f\left(x_{k}\right)-f\left(x_{k-1}\right) \geq((\alpha+\beta) / 2)\left\|x_{k}-x_{k-1}\right\|^{2} \text { for all } k \in \mathrm{N}
$$

(2) The packet sequence $\left\{f\left(x_{k}\right)\right\}$ is monotone decreasing.

(3) If $f$ is bounded from below, $g$ is $\alpha$-monotonic function and $h$ is $\beta$-monotonic function with $\alpha+\beta>0$, and $\left\{x_{k}\right\}$ is bounded, then every subsequential limit of the packets sequence $\left\{x_{k}\right\}$ is a critical point of $f$.

\subsection{Determination Sliding Window Size $W$}

In order to research the dynamic change of packet stream performance characteristics, it is very important to determine the sliding window size, because the appropriate sliding window size is helpful to analyze the encoding complexity of packet encoding and decoding performance of destination nodes. Network lifetime and power law degree distribution are important characteristics of wireless sensor networks. Many existing researches have proved that the intensity distribution and degree distribution of the Internet obey the power law distribution. Therefore, in wireless sensor networks, we use the power exponent of sensor node strength distribution and degree distribution to determine the size of the sliding window $W$.

We set the size of the sliding window to $8,16,24,32$ and 40 , respectively for experimental analysis and research. The power exponents of degree distribution is shown in Fig. 2. As can be seen from the performance analysis, when the sliding window size $W$ is smaller than 24 , the power exponent changes greatly. When the sliding window $W$ is greater than 24 , the power exponents decrease slightly.

To ensure that the sliding window has the dynamic transmission characteristics of normal data packet stream, the experimental analysis shows that the optimal size of the sliding window can be set as 24 , i.e. $\mathrm{W}=24$.

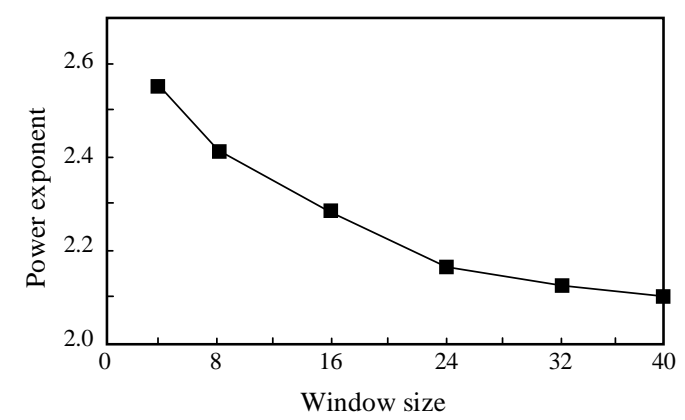

Fig. 2. Power exponent distribution of different window sizes

\section{Network Lifetime Using Network Coding}

\subsection{Network Coding Mechanism}

Linear network coding (LNC) [11] has a good coding performance. In our network coding, linear network coding is applied and improved. Several original data packets are selected for demonstration, and the linear combination of them is generated in the finite domain with 
random coefficient. The operation process of network coding packet processing is shown in Fig. 3. The encoder generates a linear combination of the currently generated raw data packets. For LNC, the coding coefficients are randomly selected in finite fields.

The middle layer represents a wireless channel in which the packet loss rate depends on the quality of the channel transmission. The encoded data packet received by the receiver is transmitted to the decoding operation, which can decode and restore the original data packet after receiving at least $g$ linearly independent data packets. In addition, instead of needing to receive all the data packets individually, the receiver can simply "keep a bucket" generated until it meets the requirements, which requires receiving more than $g$ linearly independent encoded data packets.

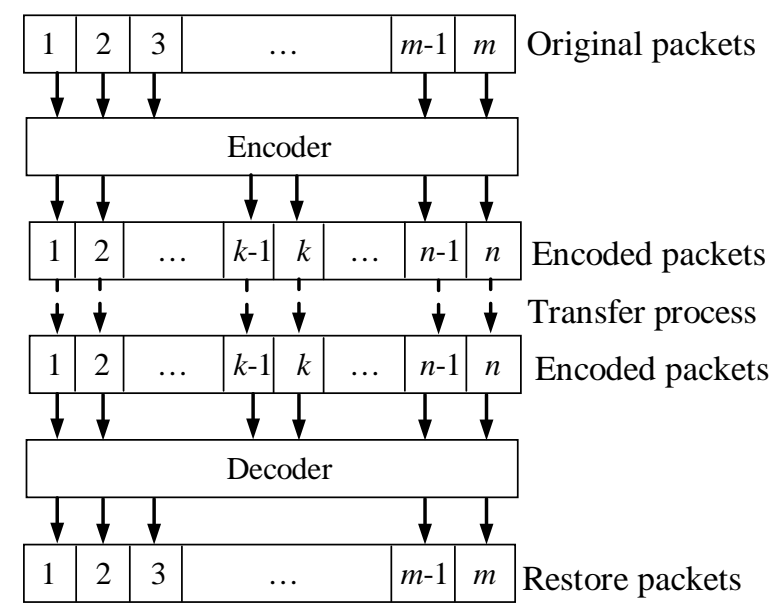

Fig. 3. Overview of network coding

\subsection{Linear Network Coding}

NC is a new coding technology, the coding nodes (intermediate nodes) in the network encode and recombine the received data packets to generate new data packets. LNC [11] is a network coding algorithm which is a linear combination of data packet received on a node and data packet sent by one or more packets. Assume that a number of original data packets $\left(p_{1}, p_{2}, \ldots\right.$, $\left.p_{n}\right)$ are generated at the source node. Let, these packets, $\left(p_{1}, p_{2}, \ldots, p_{n}\right)$, be received at an intermediate node. Suppose the intermediate node (coding nodes) can encode $v$ packets. The coding nodes will generate $n / v$ number of sliding windows, and carry out network coding for the packets in the sliding window, and then carry out data packet after transmission coding in the network.

The encoding packet using linear network encoding is regarded as an element in the finite field $G F\left(2^{s}\right)$. Here $2^{s}$ is the size of GF. In LNC, a sequence of coefficients $q=\left(q_{1}, q_{2}, \ldots, q_{n}\right)$ is picked up from $G F\left(2^{s}\right)$. The coefficients $q$ is called the network coding vector, from $G F\left(2^{s}\right)$. A set of $n$ data packets, $M_{i}(i=1,2, \ldots, n)$ is linearly network coding into a single output data packet on a coding node. The output encoded data packet consists of the following linear combinations.

$$
\mathrm{X}=\sum_{i=1}^{n} q_{i} M_{i}, \quad q_{i} \in G F\left(2^{s}\right)
$$

In addition, the network coding does not contain all data packet information for the original data packet. In the decoding node, a sufficient number $m(m \geq n)$ of decoded data packets and coefficient pairs $\left(g^{1}, X^{1}\right), \ldots,\left(g^{m}, X^{m}\right)$ are needed to restore original packets $M_{1}, \ldots, M_{n}$. In other 
words, we need to solve $m$ equations and $n$ unknowns:

$$
\mathrm{X}^{j}=\sum_{i=1}^{n} g_{i}^{j} M_{i}, \quad 1 \leq j \leq m
$$

In order to solve this equation set, the coefficients of the vectors should be linearly independent, that is, the $n \times m$ matrix should be full rank,

$$
G=\left[\begin{array}{llc}
g_{1}^{1} & \cdots & g_{1}^{j} \\
\vdots & \ddots & \vdots \\
g_{i}^{1} & \cdots & g_{i}^{j}
\end{array}\right]
$$

The original packets $M_{1}, \ldots, M_{n}$ can be found with

$$
M=G^{-1} \times X
$$

\subsection{Network Lifetime}

The network lifetime can be defined as the normal operation of all wireless sensor nodes, which is defined as follows.

In WSNs, the packets can be received for free as long as the wireless sensor nodes are deployed within the transmission range of the wireless sensor nodes, even if the packets do not fall within its transmission range. By analyzing the performance of the ALSW-NCEE's algorithm, we can derive a formula to describe the average energy consumption of the WSN.

$$
E_{\text {tot }}=E_{N C}+E_{\text {min }}+E_{E C}+E_{S W}
$$

where $E_{N C}$ represents the average energy consumption of network coding and transmission from the source node, $E_{\min }$ represents the energy consumption in the full scheduling collaboration phase, $E_{E C}$ represents the energy consumption in the competition phase, and $E_{S W}$ represents the sliding coding window in the sliding phase.

The power level of each wireless sensor node can be expressed as $P_{T}, P_{R}$ and $P_{I}$, respectively. In addition, the relation between energy and power of nodes is given by $E=P \cdot t$, where the terms $E, P$ and $t$ represent the energy parameter, the power parameter and the time parameter, respectively. Let us recall that the wireless sensor network consists of a source node, a set of $n$ intermediate nodes (code node and sliding node), and a destination node (decode node). Therefore, considering the WSN topology, we have:

$$
E_{N C S}=P_{T} \cdot T_{A}+(n+1) \cdot P_{R} \cdot T_{A}
$$

For the energy aspect of wireless sensor networks, we choose the utility function to quantify the life cycle of wireless nodes. The energy efficiency $\left(T_{i}\right)$ is evaluated as the expected number of successfully transmitted information bits $L$ divided by the total consumed energy $E_{\text {tot }}$ in the wireless sensor network, i.e.,

$$
T_{i}=\frac{L(\text { bits })}{E_{\text {tot }}(\text { Joule })}
$$

where $L$ represents the total amount of useful transmitted data packet provided and $E_{\text {tot }}$ denotes the total node energy consumed. Then the network lifetime is given by

$$
T=\min _{i \in N} T_{i}
$$

\section{Mathematical analyses}

\subsection{Probabilities to Packet Loss}

Through network coding, data packets are passed to the application in a sliding window. For each sliding coding packet, the receiving node needs to receive $B$ independent coding packets, 
so as to better decode the received packets and restore the original data packets. Let $N_{i}$ represent the number of sending network coded data packets until the receiving node decodes the encoded data packet i. $p$ represents the packet loss rate. Aiming at the binomial distribution mechanism, the probability that the receiver can accurately receive $B$ coding packets after the sender transmits the coded data packet $i$ for the $n$-th time is given.

$$
P\left\{N_{i}=n\right\}=\left(\begin{array}{c}
n-1 \\
B-1
\end{array}\right)(1-p)^{B} p^{n-B}, n \geq B
$$

This is the probability that the $B-1$ data packet in the first $n-1$ transmission will be successfully received by the receiving node and the last transmission will be successful. Recall that for now we assume that all network coded data packets are independent, following the encoding mechanism described earlier. So we get,

$$
\begin{aligned}
P\left\{N_{i} \leq n\right\} & =\sum_{i=B}^{n}\left(\begin{array}{c}
i-1 \\
B-1
\end{array}\right)(1-p)^{B} p^{i-B} \\
& =(1-p)^{B} \sum_{i=0}^{n-B}\left(\begin{array}{c}
B+i-1 \\
B-1
\end{array}\right) p^{i}
\end{aligned}
$$

\subsection{Assign Probabilities to Sliding Windows}

Once the sliding window boundary is set in the data packet set to be re-encoded, the sliding process can assign a probability to each sliding window according to the window information contained in the sliding window.

$P\left(d_{p, w} \mid s\right)$, the sliding window size of $w$ packets either side of $p$, and the correlation probability of $\mathrm{d}$ in $p$ position given the packets returned by the input system $s$ is

$$
P\left(d_{p, w} \mid s\right)=\frac{\sum_{i=f}^{e} P\left(d_{i} \mid s\right)}{e-f+1}
$$

Using a sliding window reduces the probability behind the result set more smoothly than using only the probability based on the data packet available at each location.

\section{Simulation Experiments}

\subsection{Simulation Parameters}

In this section, we present some simulation experiment results of the proposed a Network Coding-based Maximum Lifetime algorithm for Sliding Window in WSNs (NC-MLSW), which improves the network throughput, network lifetime and packet loss probability of WSN. In our simulations, the area of the WSN is $1000 \mathrm{~m} \times 1000 \mathrm{~m}$, the number of sensor nodes is 100 , the wireless network bandwidth is $5 \mathrm{Mb} / \mathrm{s}$, the packets queue size is 100 packets. Each packet is $1000 \mathrm{~B}$ and each ACK is $40 \mathrm{~B}$. The proposed algorithm was compared with MWNCast algorithm [13] and OQMCAR [20] in WSN environment. The corresponding simulation experiment parameters are summarized in Table 1.

In this section, we use a randomly generated network to execute the algorithm [26]. Simulation experiment results show that the method has good network performance evaluation. We use the network simulation software (NS-2) simulator [27] to evaluate the NC-MLSW in WSNs. NS-2 is a discrete event simulation software for network technology research. NS-2 provides extensive support and performance comparisons for TCP, network coding, and energy efficiency in wired and wireless networks. 
Table 1. Simulation Experiment Parameters

\begin{tabular}{|c|c|}
\hline Parameter & Range \\
\hline \hline Number of sensor nodes & 100 \\
\hline WSN area & $1000 \mathrm{~m} \times 1000 \mathrm{~m}$ \\
\hline Transmission range & $250 \mathrm{~m}$ \\
\hline Simulation time & 600 seconds \\
\hline Beacon period & $100 \mathrm{~ms}$ \\
\hline Communication model & Constant bit rate (CBR) \\
\hline Window size & W=24 \\
\hline Message size & 512 bytes/packet \\
\hline Examined routing protocol & MWNCast [13], OQMCAR [20] \\
\hline
\end{tabular}

\subsection{Simulation Results}

Now we analyze the performance of three algorithms in wireless sensor networks. We study the tradeoff between the network throughput, network lifetime, and packet loss probability for a network with 100 nodes, the window size of NC-MLSW is set to 16 .

In Fig. 4, we analyzed the data packet throughput of NC-MLSW, MWNCAST and OQMCAR in the wireless sensor. It can be seen from the performance analysis that the data packet throughput of NC-MLSW algorithm is superior to MWNCAST algorithm and OQMCAR algorithm. It can also be seen that the data packet throughput of NC-MLSW algorithm increases with the increase of the number of network nodes, which also shows better performance. Therefore, we can see that by taking advantage of network coding, NC-MLSW algorithm is effective in improving the data packet throughput.

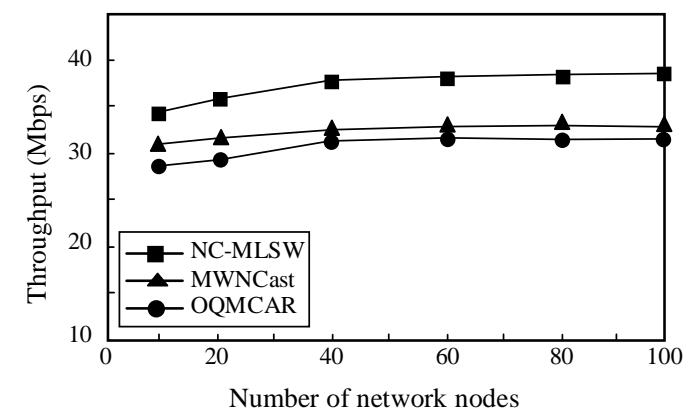

Fig. 4. Network packet throughput vs. Number of network nodes

The average network lifetime has been depicted in Fig. 5. As the number of network nodes increases, so does the lifetime of the network, because more node data and data packets are active in each node's transport region. As a result, the amount of data involved in coding nodes in wireless network is reduced and the network coding ability of wireless nodes is improved. However, the network lifetime in the NC-MLSW approach increases and remain constant despite of the increase in number of wireless nodes because packets are recovered from the encoded packets and the decoded data packet on the destination node.

Fig. 6 shows that the packet loss probability of data packet transmission is increase dramatically since the increase the number of network sensor nodes. The NC-MLSW algorithm has lower packet loss probability. We can find from Fig. 6 that the packet loss probability curve of NC-MLSW is below those of other two algorithms. From the Fig. 6, with the increase of network sensor nodes, the packet loss rate increases almost exponentially. 


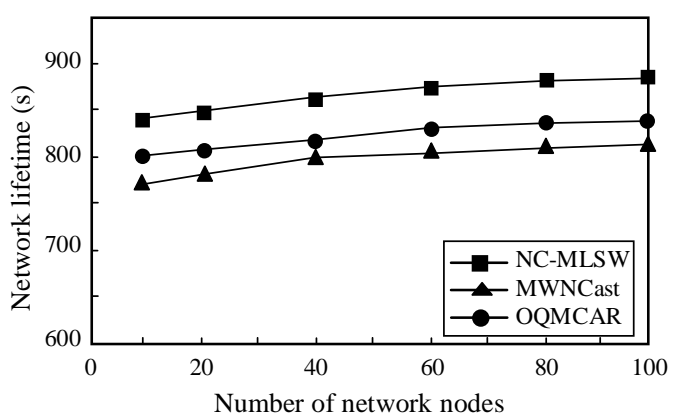

Fig. 5. Network lifetime vs. Number of network sensor nodes

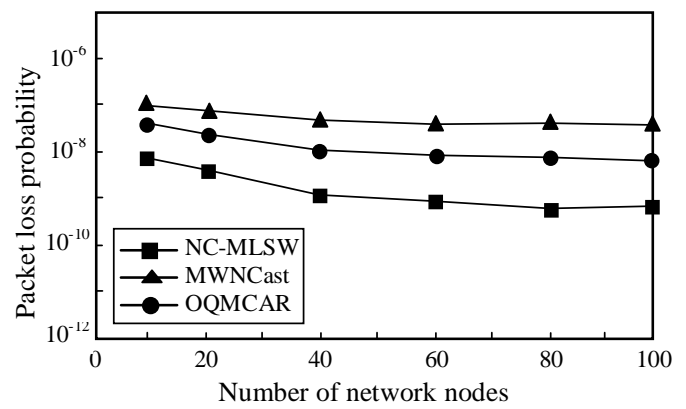

Fig. 6. Packet loss probability vs. Number of network sensor nodes

\section{Conclusion}

In this paper, the performance of wireless sensor network is improved by combining sliding window and network coding. We present a Network Coding-based Maximum Lifetime algorithm for Sliding Window in WSNs (NC-MLSW). Then, theoretical analysis is presented to verify the better performance of proposed MLNC-SW algorithm. By applying network coding and intermediate node to network coding of data packet in sliding window, this algorithm can improve network throughput. The analysis shows that MLNC-SW produces higher network throughput, prolongs network lifetime, and lowers packet loss probability. This algorithm not only guarantees the reliability of data transmission, but also increases the lifetime of the network, improves the performance of node encoding/decoding, reduces the complexity of encoding/decoding, and provides good support for the application of wireless sensor network.

\section{References}

[1] R. Ahlswede, N. Cai, S.-Y.R. Li, R. W. Yeung, "Network information flow," IEEE Transactions on Information Theory, vol. 46, no. 4, pp. 1204-1216, 2000. Article (CrossRef Link)

[2] O. B. Rhaiem, L. Chaari, "Information transmission based on network coding over wireless networks: a survey,” Telecommunication Systems, vol. 65, no. 4, pp. 551-565, 2017. Article (CrossRef Link)

[3] R. W. Yeung, "Network coding: A historical perspective," Proceedings of the IEEE, vol. 99, no. 3, pp. 366-371, 2011. Article (CrossRef Link) 
[4] F. Li, W. M. Guo, “An Efficient Polynomial Time Algorithm for Robust Multicast Network Code Construction,” IEEE Communications Letters, vol. 19, no. 2, pp. 143-146, 2015. Article (CrossRef Link)

[5] T. Aktas, Y. A. Ozgür, E. Aktas, "Practical methods for wireless network coding with multiple unicast transmissions,” IEEE Transactions on Communications, vol. 61, no. 3, pp. 1123-1133, 2013. Article (CrossRef Link)

[6] Y. S. Wang, W.-S. Yang, J. Wu, "Analysis of a Hypercube-Based Social Feature Multipath Routing in Delay Tolerant Networks," IEEE Transactions on Parallel and Distributed Systems, vol. 24, no. 9, pp. 1706-1716, 2013. Article (CrossRef Link)

[7] B. L. Sun, C. Gui, Y. Song, and H. Chen, “A Novel Network Coding and Multi-path Routing Approach for Wireless Sensor Network,” Wireless Personal Communications, vol. 77, no. 1, pp. 87-99, 2014. Article (CrossRef Link)

[8] B. L. Sun, C. Gui, Y. Song, H. Chen, X. Y. Zhu, "Performance Analysis of Sliding Window Network Coding in MANET," 11th Conference on Advanced Computer Architecture, Weihai, China, pp. 174-183, August 22-23, 2016. Article (CrossRef Link)

[9] R. R. Rout, and S. K. Ghosh, "Enhancement of Lifetime using Duty Cycle and Network Coding in Wireless Sensor Networks,” IEEE Transactions on Wireless Communications, vol. 12, no. 2, pp. 656-667, 2013. Article (CrossRef Link)

[10] K.-H. Lee, J.-H. Kim, S. Cho, "Power saving mechanism with network coding in the bottleneck zone of multimedia sensor networks,” Computer Networks, vol. 96, pp. 58-68, 2016. Article (CrossRef Link)

[11] Y. Gu, H. Han, X. J. Li, J. Guo, "Network Coding-Aware Routing Protocol in Wireless Mesh Networks,” Tsinghua Science and Technology, vol. 20, no. 1, pp. 40-49, Feb. 2015. Article (CrossRef Link)

[12] Haiqiang Chen, Yunyi Liu, Tuanfa Qin, Haitao Yao, and Qiuling Tang. Construction of Structured q-ary LDPC Codes over Small Fields Using Sliding-Window Method," Journal of Communications and Networks, vol. 16, no. 5, pp. 479-484, 2014. Article (CrossRef Link)

[13] Fei Wu, Cunqing Hua, Hangguan Shan, Aiping Huang, "Cooperative multicast with moving window network coding in wireless networks,” Ad Hoc Networks, vol. 25, no. A, pp. 213-227, 2015. Article (CrossRef Link)

[14] X. Li, C.-C. Wang, X. Lin, "On the capacity of immediately-decodable coding schemes for wireless stored-video broadcast with hard deadline constraints," IEEE Journal on Selected Areas in Communications, vol. 29, no. 5, pp. 1094-1105, 2011. Article (CrossRef Link)

[15] J. Liu, Y. Zhu, G. X. Kang, Y. F. Zhang, “Optimal Energy-Efficient Power Allocation and Outage Performance Analysis for Cognitive Multi-Antenna Relay Network Using Physical-Layer Network Coding," KSII Transactions on Internet and Information Systems, vol. 7, no. 12, pp. 3018-3036, 2013. Article (CrossRef Link)

[16] J. Tian, T. Yan, and G. L. Wang, “A Network Coding Based Energy Efficient Data Backup in Survivability-Heterogeneous Sensor Networks,” IEEE Transactions on Mobile Computing, vol. 14, no. 10, pp. 1992-2006, 2015. Article (CrossRef Link)

[17] A. Fiandrotti, V. Bioglio, M. Grangetto, R. Gaeta, and E. Magli, "Band Codes for Energy-Efficient Network Coding With Application to P2P Mobile Streaming,” IEEE Transactions on Multimedia, vol. 16, no. 2, pp. 521-532, 2014. Article (CrossRef Link)

[18] Y. Zhou, L. H. Yang, L. X. Yang, "Improved Compressed Network Coding Scheme for Energy-Efficient Data Communication in Wireless Sensor Networks,” KSII Transactions on Internet and Information Systems, vol. 11, no. 6, pp. 2946-2962, 2017. Article (CrossRef Link)

[19] L. H. Ding, P. Wu, H. Wang, Z. W. Pan, and X. H. You, "Lifetime maximization routing with network coding in wireless multihop networks,” Science China (Information Sciences). Vol. 56, no. 2, pp 1-15, 2013. Article (CrossRef Link)

[20] C. B. Lu, S. Xiao, Y. B. Miao, “OQMCAR: An enhanced network coding-aware routing algorithm based on queue state and local topology,” KSII Transactions on Internet and Information Systems, vol. 9, no. 8, pp. 2875-2893, 2015. Article (CrossRef Link) 
[21] J. Wang, M. S. Ouyang, W. Liang, J. Hou, X. Y. Liu, "Device-to-Device Relay Cooperative Transmission Based on Network Coding," KSII Transactions on Internet and Information Systems, vol. 11, no. 7, pp. 3431-3445, 2017. Article (CrossRef Link)

[22] C. H. Gebotys, B. A. White, "A Sliding Window Phase-Only Correlation Method for Side-Channel Alignment in a Smartphone," ACM Transactions on Embedded Computing Systems, vol. 14, no. 4, Article 80, 22 pages, 2015. Article (CrossRef Link)

[23] G. Stitt, E. Schwartz, P. Cooke, "A Parallel Sliding-Window Generator for High-Performance Digital-Signal Processing on FPGAs," ACM Transactions on Reconfigurable Technology and Systems, vol. 9, no. 3, Article 23, 22 pages, 2016. Article (CrossRef Link)

[24] L. S. Tan, M. Wu, "Data reduction in wireless sensor networks: a hierarchical LMS prediction approach,” IEEE Sensors Journal, vol. 16, no. 6, pp. 1708-1715, 2016. Article (CrossRef Link)

[25] L. S. Tan, S. D. Tang, "Energy Harvesting Wireless Sensor Node With Temporal Death: Novel Models and Analyses," IEEE/ACM Transactions on Networking, Vol. 25, No. 2, pp. 896-909, 2017. Article (CrossRef Link)

[26] B. Waxman, "Routing of Multipoint Connections," IEEE Journal on Selected Areas in Communications, vol. 6, no. 9, pp. 1617-1622, 1988.

[27] The Network Simulator - NS-2, http://www.isi.edu/nsnam/ns/.

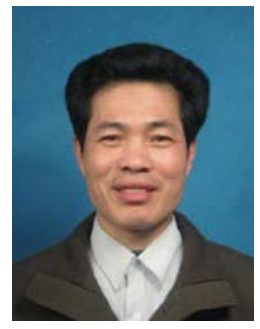

Baolin Sun received his PhD degree in computer science and technology in 2006 and a master degree of computer science and technology in 1999 from the Wuhan University of Technology, China. He is currently a professor at the school of information and engineering in the Hubei University of Economics, Wuhan, China. He was awarded the Province Special Prize by the Hubei Province Government in 2007. He is an IAENG member, and one of Editorial Board Guest Members of World Sci-Tech R\&D, and also an International Standard Draft organizing members of ISO/IEC JTC1/SC6. His research interests include multipath routing, parallel and distributed computing, network optimization and ad hoc networks. He has published over 150 journal and conference papers and has author of four books in the above areas.

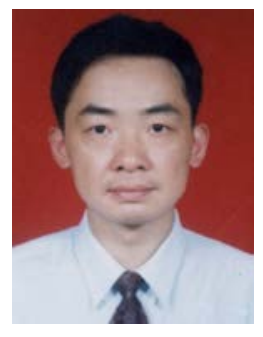

Chao Gui received his master degree of computer science and technology in 1989 from the Wuhan University, Wuhan, China. He is currently a professor at the school of information and engineering in the Hubei University of Economics, Wuhan, China. His research interests include wireless communication, performance analysis and modeling. He has published over 60 research papers.

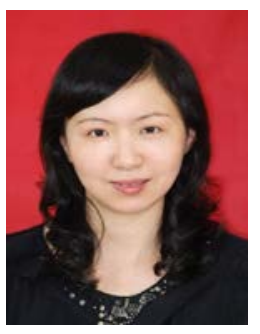

Ying Song received her $\mathrm{PhD}$ degree in photogrammetry and remote sensing in 2011 and a master degree of geographical information system in 2004 from the Wuhan University, Wuhan, China. She is currently an associate professor at the school of information and engineering in the Hubei University of Economics, Wuhan, China. Her research interests include wireless communication, mesh networks and network protocol. She has published over 30 research papers.

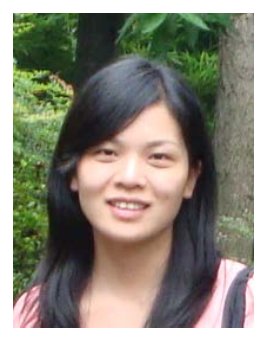

Hua Chen received her master degree of computer science and technology in 2005 from the Huazhong University of Science and Technology, Wuhan, China. Her research interests include algorithm, wireless communication, performance analysis, and network model. She has published over 20 research papers. 